\title{
Adipose Stem Cell Coating of Biomimetic $\beta$-TCP Macrospheres by Use of Laboratory Centrifuge
}

\author{
Joshua Chou, David W. Green, ${ }^{2}$ Krishneel Singh, Jia Hao, ${ }^{1}$ Besim Ben-Nissan,, and Bruce Milthorpe ${ }^{1}$
}

\begin{abstract}
Biomimetic materials such as coral exoskeletons possess unique architectural structures with a uniform and interconnected porous network that can be beneficial as a scaffold material. In addition, these marine structures can be hydrothermally converted to calcium phosphates, while retaining the original structural properties. The ability of biomaterials to stimulate the local microenvironment is one of the main focuses in tissue engineering, and directly coating the scaffold with stem cells facilitates future potential applications in therapeutics and regenerative medicine. In this article we describe a new and simple method that uses a laboratory centrifuge to coat hydrothermally derived beta-tricalcium phosphate macrospheres from coral exoskeleton with stem cells. In this research the optimal seeding duration and speed were determined to be $1 \mathrm{~min}$ and $700 \mathrm{~g}$. Scanning electron micrographs showed complete surface coverage by stem cells within 7 days of seeding. This study constitutes an important step toward achieving functional tissue-engineered implants by increasing our understanding of the influence of dynamic parameters on the efficiency and distribution of stem cell attachment to biomimetic materials and how stem cells interact with biomimetic materials.
\end{abstract}

Key words: adipose-derived stem cells; biomimetic scaffolds; cell seeding; scaffold coating; stem cell coating

\section{Introduction}

T HE DEVELOPMENT OF BIOMATERIALs, combined with advances in tissue engineering, is yielding promising results for repair, replacement, or modification of various tissues and organs. ${ }^{1}$ Scaffold materials, depending on the desired applications, generally vary in their compositional makeup, but the general consensus of developing more bioactive scaffolds remains unchanged. Enhancing the bioactivity of the scaffold would allow for faster engineered tissue generation and recovery period. Coral exoskeletons have been successfully used as biomaterial constructs and can be hydrothermally converted ${ }^{2}$ to biocompatible calcium phosphates while retaining architectural structure. ${ }^{3}$ Specifically, Foraminifera exoskeletons possess an interconnected and uniform porous network and have been shown to stimulate osteoblast proliferation while reducing monocyte activities. ${ }^{4}$ With the continual development in the application of stem cells in tissue engineering, it is not difficult to envision the potential therapeutic efficacy of combining biomimetic materials with stem cells. Cells isolated from the host patient onto the scaffold would need to be seeded onto the scaffold construct. The success of cell seeding depends on the efficiency, the time required for the cells to attach to the scaffold, the viabil- ity of cells, and the uniform spatial distribution of the cells throughout the scaffold. One of the key factors affecting the efficiency of cell seeding is the morphology and architecture of the scaffold material, which plays an important role in cell attachment and viability. Studies have shown, for example, that smaller scaffold pore diameters $(\sim 30 \mu \mathrm{m})$ enhance cell-seeding efficiency ${ }^{5,6}$ compared with larger pore sizes $(\sim 70 \mu \mathrm{m})$. It is also crucial to try to achieve a uniform cell distribution throughout the scaffold because this will allow uniform tissue growth, while limiting any adverse effects from contact inhibition. ${ }^{7}$ Furthermore, since stem cells are anchorage dependent, the faster the cells are able to attach to the scaffold during the cell seeding process, the more tissue growth will be accelerated and cell viability enhanced. Several techniques are currently used for seeding cells onto scaffold, and this can include simply seeding the cells directly onto the scaffold material and allowing the cells to grow over time, preferably undisturbed, with the expectation of cellular attachment and migration onto the scaffolds. ${ }^{8}$ More dynamic techniques can include a setup similar to bioreactors in which there is a constant flow of media; such a setup features oscillatory pressure through the scaffold material after the cells have been seeded. ${ }^{9,10}$ These methods can achieve the intended goal; however, they are limited by the amount

\footnotetext{
${ }^{1}$ Advanced Tissue Regeneration and Drug Delivery Group, School of Medical and Molecular Biosciences, University of Technology Sydney, Sydney, Australia.

${ }^{2}$ Faculty of Dentistry, Prince Philip Dental Hospital, The University of Hong Kong, Hong Kong, China.
} 
of time required for seeding and often require high concentrations of cells because not all the cells can completely adhere to the material and there is an initial loss of cell viability.

During the last decade a number of problems were observed that restricted the use of stem cells in the physiologic environment. The best type of therapeutic stem cells are still not known, the number of stem cells that can be isolated from a single patient is insufficient, not enough stem cells can be kept alive in cultivation, and the tissues become damaged during transplantation. These facts highlight the need for better practices and procedures in stem cell cultivation and targeted placement. Increasingly, there is also a need for better and simpler placement or coating processes and biomaterials and devices with which to process and guide these cells into functional tissues. ${ }^{11}$

In this article we focus on the use of a common laboratory centrifuge to facilitate the transfer of stem cells into porous scaffolds, which provide a quick, simple, and functional method for stem cell incorporation and coating.

\section{Materials and Methods}

\section{Scaffold preparation}

The scaffold material was prepared by using marine exoskeletons (Foraminifera), hydrothermally converting them to beta-tricalcium phosphate $(\beta$-TCP), and characterizing them according to previously published methodology. ${ }^{4}$ In brief, Foraminifera material was cleaned in sodium hydrochlorite and subject to hydrothermal conversion at $220^{\circ} \mathrm{C}$ by means of chemical exchange between ammonium hydrogenphosphate (Sigma Aldrich, Sydney, Australia).

\section{Isolation and culture of adipose-derived stem cells}

Adult Fischer rats (Rattus norvegicus) were used for the isolation of rat adipose-derived stem cells (ADSCs). Animals were housed and cared for under standard conditions and were euthanized in a $\mathrm{CO}_{2}$ chamber. The inguinal fat pad was then harvested, and ADSCs were isolated. All subsequent steps were conducted under sterile conditions. The harvesting methods for adipose tissue used followed established protocol and methods by Bunnell et al. ${ }^{12}$ Fat pads were rinsed twice in Dulbecco's modified Eagle's medium (DMEM; Gibco). The fat pad was then minced with a pair of scissors to form a fine slurry, which was then digested in collagenase type 1 (Gibco) for $45 \mathrm{~min}$ at $37^{\circ} \mathrm{C}$. The suspension was centrifuged at $1600 \mathrm{~g}$ for $10 \mathrm{~min}$ at $4^{\circ} \mathrm{C}$ to separate adipocytes by the addition of Ficoll to separate and remove the interface between the Ficoll and DMEM. This was then resuspended in $8 \mathrm{~mL}$ of DMEM to dilute any residual Ficoll. The cells were washed twice in DMEM and centrifuged at $1000 \mathrm{~g}$. Upon completion of the final wash the pellet was resuspended in basal growth media DMEM Glutmax/F12 (Gibco) with $10 \%$ fetal bovine serum (FBS, Invitrogen) and 1\% antibiotics/antimycotics (ABAM; Invitrogen). Two-milliliter aliquots of the suspension were placed in T25 culture flasks (Nunc) and incubated at $37^{\circ} \mathrm{C}$ at $5 \% \mathrm{CO}_{2}$ for 2 days until ADSCs adhered to the culture flask. Nonadherent cells were eliminated by aspirating floating cells and replacing the media. ADSCs were passaged three times before use in experiments.

Rat adipose tissue-derived stem cells were grown in medium consisting of DMEM (Invitrogen) containing $1 \mathrm{~g} / \mathrm{L}$ glu- cose, supplemented with $10 \%$ FBS, $100 \mathrm{U} / \mathrm{mL}$ penicillin, and $100 \mathrm{mg} / \mathrm{mL}$ streptomycin sulfate. The cells were incubated in a humidified, $37^{\circ} \mathrm{C}, 5 \% \mathrm{CO}_{2}$ incubator, with growth medium being replaced every 3-4 days. The cells were expanded to $80 \%-90 \%$ confluence prior to trypsinization for passage or scaffold seeding.

\section{Stem cell coating of scaffold}

The scaffold material, measuring $1.4 \mathrm{~mm}$ in diameter, was coated individually by separately placing each sphere in a 15$\mathrm{mL}$ Falcon tube containing $1 \mathrm{~mL}$ of the cell suspension $\left(6 \times 10^{6}\right.$ cells). The tubes were placed in a laboratory centrifuge (Eppendorf Centrifuge 5702) for 1, 2, and $3 \mathrm{~min}$ at $700 \mathrm{~g}$. Following this the cap of the tubes were released slightly and placed back in a $37^{\circ} \mathrm{C}$ incubator with $5 \% \mathrm{CO}_{2}$. After $48 \mathrm{~h}$ to allow the cells to adhere, the scaffolds from each tube were carefully transferred back into a 24-well plate with fresh media for 3, 5, and 7 days.

\section{Protein quantification}

At each time point, the scaffolds were first washed three times with PBS and transferred to 1.5-mL Eppendorf tubes with $300 \mu \mathrm{L}$ of Triton X-100 lysis buffer for $24 \mathrm{~h}$. The protein content from the cell lysate was determined by BioRad DC Protein assay kit according to the manufacturer's instructions $(10 \mu \mathrm{L}$ of the lysate with $25 \mu \mathrm{L}$ of Reagent $\mathrm{A}$ and $200 \mu \mathrm{L}$ of Reagent B). Protein standards were made with various concentrations of bovine serum albumin. The resulting values were compared with a standard curve, which was generated for each separate experiment with a fresh set of standards.

\section{Cell adherence and cell viability}

After 7 days of culture, the media was removed and washed three times with PBS, and $500 \mu \mathrm{L}$ of TrypLe was added for $10 \mathrm{~min}$ followed by the addition of $1 \mathrm{~mL}$ of ADSC media to neutralize the TrypLe reaction. Following this, $100 \mu \mathrm{L}$ of the media was extracted and $1 \mu \mathrm{L}$ of propidium iodide was added for $10 \mathrm{~min}$. The ADSC viability and the respective number of cells attached to the scaffold were assessed by Invitrogen Tali ${ }^{\mathrm{TM}}$ Cytophotometer and in accordance to manufacturer's instructions and guidelines.

\section{Scanning electron microscopy}

At each predetermined time point, the medium was removed and the scaffolds were immersed in $300 \mu \mathrm{L}$ of $4 \%$ formaldehyde for $1 \mathrm{~h}$ followed by serial graduation of ethanol $(60 \%, 70 \%, 80 \%, 90 \%$, and $100 \%)$. The scaffolds were subsequently air-dried for $24 \mathrm{~h}$ before being characterized by a Zeiss Supra 55VP scanning electron microscope coupled with a back scattered electron detector under variable pressure at $2.2 \times 10^{6}$ Torr.

\section{Statistical analysis}

All values are presented as the mean \pm standard deviation. All data were examined based on three to five different measurement values. The data were analyzed by single-factor analysis of variance (ANOVA) in which significantly different pairs were defined as having $p<0.05$. All samples were measured in triplicate, and the experiment was repeated three times. Metric data were analyzed by one-way ANOVA followed by Scheffe's post hoc test at a significance 


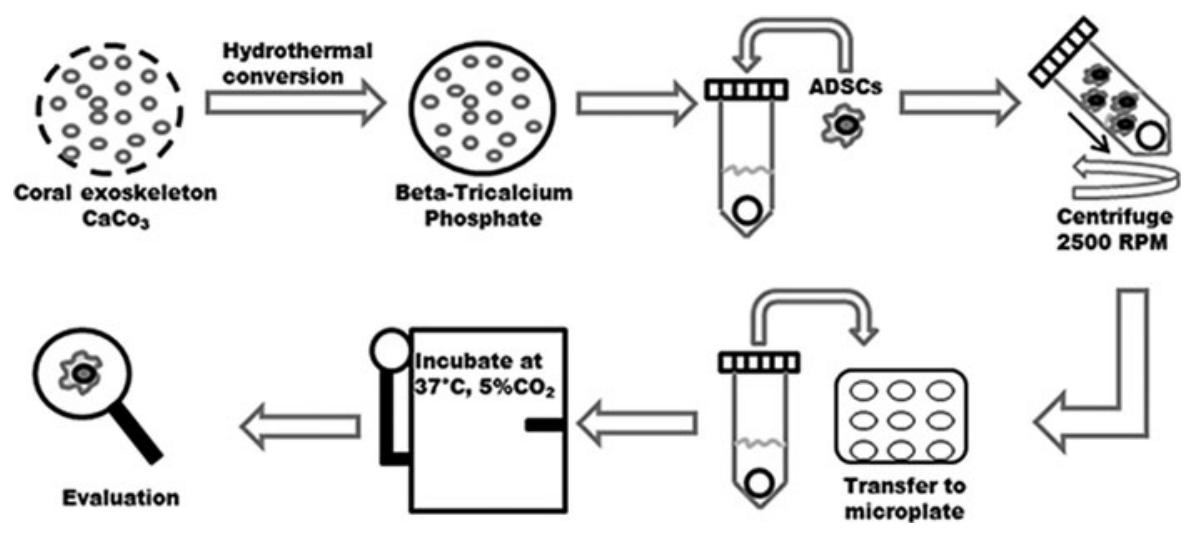

FIG. 1. A schematic diagram detailing the experimental setup from conversion of the scaffold material to the coating of the adipose-derived stem cells (ADSCs); $2500 \mathrm{rpm}=700 \mathrm{~g}$. level of 0.05 . For the protein analysis, the samples were loaded in triplicate and standardized with the plate blank.

\section{Results}

\section{Optimization of ADSC coating}

A schematic diagram of the experimental setup is shown in Fig. 1. The centrifuge times tested included 1, 2, and $3 \mathrm{~min}$, after which the scaffolds were grown for 5 days before cellular protein analysis. The cellular protein content from the scaffolds was used to indicate the number of cells coated onto the scaffolds. As a mean of standardizing, protein content was subtracted with the amount of protein found in cell-free macrosphere scaffolds. The protein content presented in Fig. 2 showed that 1-min coating provided the most optimal cell seeding on the $\beta$-TCP macrospheres. There was a significantly greater amount of cellular protein from the cells from the 1-min centrifuge time compared with longer duration of spinning and with static seeding. With 2- and 3-min centrifugation, cells were still able to be seeded on the scaffold but at a significantly lower number compared with the 1-min centrifugation.

\section{Protein and cellular analysis}

Since 1-min centrifugation produced the most efficient results, subsequent experiments and characterization were

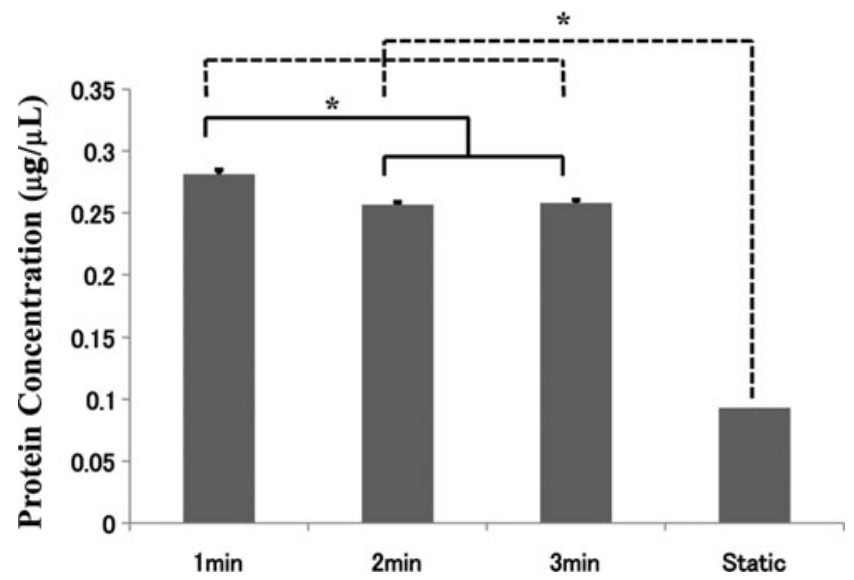

FIG. 2. Seeding of ADSCs on the scaffold after 1, 2, and $3 \mathrm{~min}$ of centrifugation and static seeding. One-minute centrifugation resulted in the highest cellular protein concentration. *Statistically significant $(p<0.05)$. based on the 1-min coating time. Figure 3 shows the approximate numbers of cells that were seeded over 3, 5, and 7 days, and Fig. 4 shows the associated cell viability. A significant increase in cell number occurred over the 7-day period, with an approximate cell number of $1 \times 10^{4}$ cells $/ \mathrm{mL}$ seeded onto the scaffold, utilizing $2 \times 10^{6}$ cells initially. The cell viability also showed an increase over the 7-day period and rose from approximately $78 \%$ to $95 \%$. The cellular proteins were also determined over the same experimental period of 3,5 , and 7 days, and the results are presented in Fig. 5. The results show once again that the cellular proteins increased over the 7-day period, which reinforces and complements the results for the number of cells that were seeded onto the scaffold.

\section{Scanning electron imaging of ADSC coating}

In addition to being able to seed a significant number of ADSCs onto the scaffold, achieving homogeneity in the cellular distribution is also important. Scanning electron microscopy images were taken at 3 and 7 days to determine the distribution of the cells on the scaffolds. Figure $6 \mathrm{a}-\mathrm{c}$ shows the cellular distribution at 3 days, and it can be seen that the cells not only adhered to the scaffold but were also distributed among the pores and throughout the surface of the scaffold. Figure $6 \mathrm{~d}-\mathrm{f}$ shows that after 7 days, the cells were able to coat the majority of the scaffold, and a significant difference could be seen compared with 3 days.

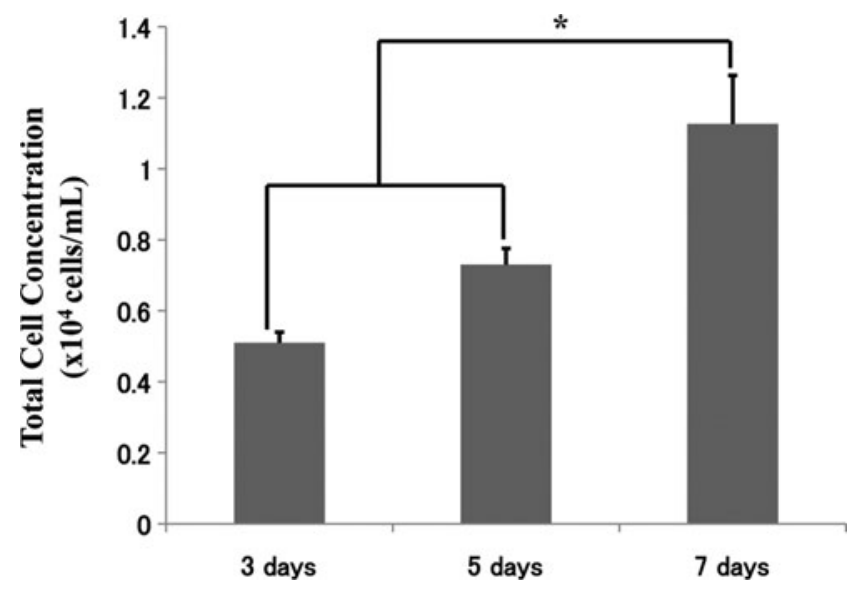

FIG. 3. The number of ADSCs attached to the $\beta$-TCP macrospheres after 3,5 , and 7 days, showing continual cell growth over this period time. ${ }^{*}$ Statistically significant $(p<0.05)$. 


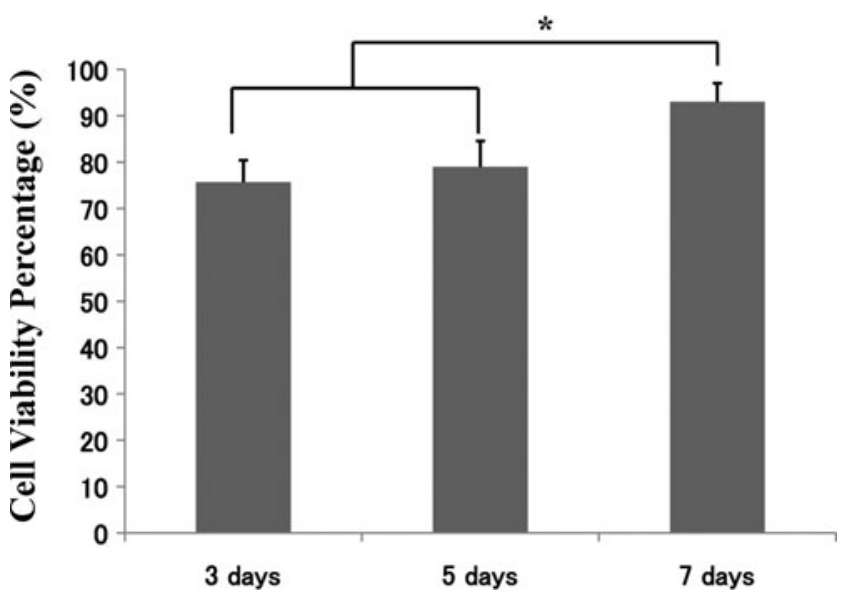

FIG. 4. The associated cell viability between 3 and 7 days from Fig. 2, showing increase cell viability. *Statistically significant $(p<0.05)$.

\section{Discussion}

In the development of suitable biomaterials for tissue engineering applications, one of the fundamental goals is stimulating the local environment surrounding the scaffold. With the emergence of stem cells and their wide array of applications in tissue engineering, many successful techniques have been developed over the years to introduce cells into biomaterials. Unfortunately seeding efficiencies have not yet reached an optimal level in which low-density cells can be seeded and be ready for implantation in a limited amount of time. The aim of this research was to determine whether marine exoskeleton that has been hydrothermally converted to $\beta$-TCP can be coated with ADSCs to improve its therapeutic efficiency. The interest in using marine exoskeleton arises from its unique architectural structure which has advantages as a scaffold matrix for bone ingrowth. In addition, by hydro-

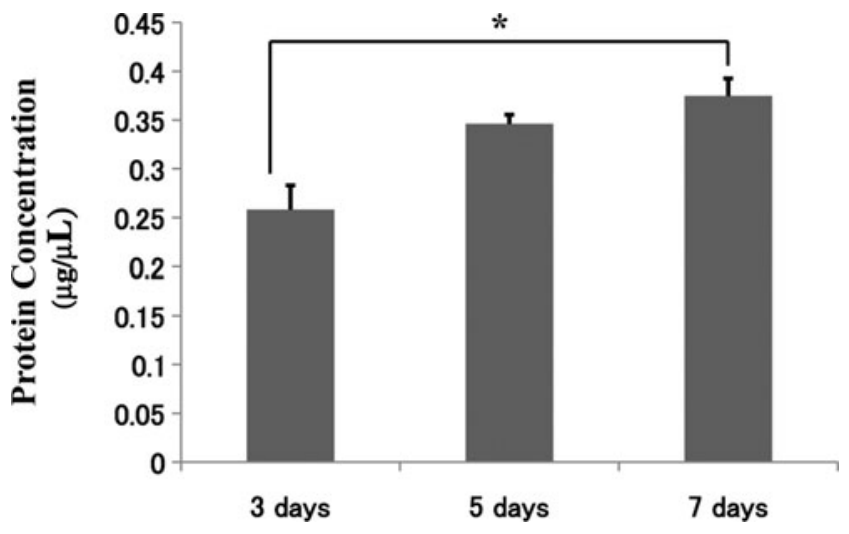

FIG. 5. Cellular protein analysis after ADSC coating at 3, 5, and 7 days, showing continual growth of the cells on the scaffold. *Statistically significant $(p<0.05)$.

thermal conversion, the calcium carbonate exoskeleton can be easily converted to various derivatives of calcium phosphate. By using a laboratory centrifuge to coat the scaffold with ADSCs, this technique can provide a quick and easy method for coating other biomaterials with ADSCs.

To improve the efficiency of the ADSC coating on the $\beta$-TCP macrospheres, initial experiments were done to determine the optimal duration for seeding the cells on the scaffold. The rotational speed of the centrifuge was set at $700 \mathrm{~g}$ because the harmonics of the centrifuge prevented smooth rotation at speeds below $448 \mathrm{~g}$, which would result in cell shearing and possible cell damage. ${ }^{13}$ With higher rotational speed, this would increase the possibility of the cells being spun down too fast and limit the amount of cells that can be seeded onto the scaffold. From the initial optimization process it was found that 1-min centrifugation seeded the greatest number of cells onto the scaffold. Increasing the centrifugation time to 2 and $3 \mathrm{~min}$ seeded the cells at a significantly lower
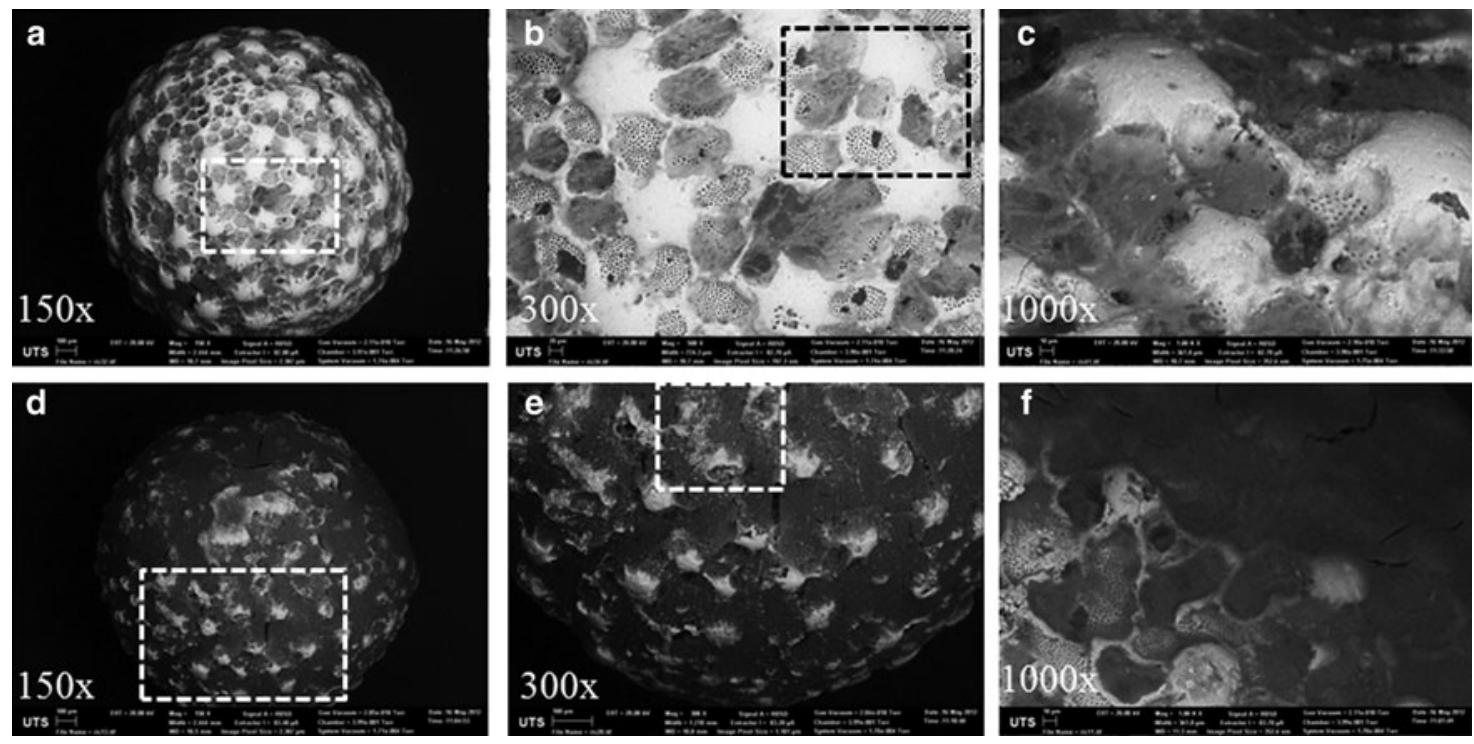

FIG. 6. Scanning electron microscopy images showing ADSC-seeded macrospheres after 3 days (a-c) showing ADSCs growing in the surface crater and spreading across the material. After 7 days of seeding (d-f), ADSCs completely covered the scaffold material. 
concentration. We hypothesize that with increasing time, the cells are completely pushed through the scaffold or around it to the bottom of the tube. Therefore increasing the centrifugation time would not increase the likelihood of more cells being seeded onto the scaffold but rather increase the risk of damaging some of the cells. As such, by using 1-min centrifugation, the $\beta$-TCP macrospheres were evaluated over a 7 -day period. Even though it was confirmed that ADSCs can be seeded onto the scaffold, it was equally important to determine how these ADSCs behaved after being seeded and whether they would continue to proliferate or eventually become apoptotic. During the 7-day experimental period the results showed that both the cell numbers and cellular proteins were increased, indicating the ADSCs were seeded successfully and were proliferating on the scaffold material. In addition to this, cell viability results showed an initial viability of $78 \%$ and this was increased to $95 \%$ at 7 days, which affirmed that the cells were viable and are capable of inducing and stimulating the surrounding local environment once implanted. Furthermore, coating the scaffold through using the centrifuge allowed homogenous seeding, which can reduce the amount of time needed to prepare scaffolds for implantation. From the scanning electron microscopy images presented in Fig. 6, it can be seen that after 3 days the ADSCs were seeded within the pore craters of the macrospheres and were homogenously distributed. While most of the pore craters were covered by the ADSCs, there remained some pore craters that still had visible exposed pores. However, at 7 day there was complete coverage of the ADSCs on the $\beta$-TCP macrospheres. It is important to note that depending on the intended application, a confluent coating of the scaffold might not be ideal because this can prevent migration of other crucial cells ${ }^{14}$ and as such, the confluency of the coating can be controlled and tailored to a specific application by limiting the growth period. It is envisaged that the combined effect of the stem cell coating with the biomimetic $\beta$-TCP spheres can be applied in non-load-bearing implant applications. As previously mentioned, the scaffold construct possesses uniform and interconnected pores providing an ideal environment for improved bone ingrowth and vascularization. Combined with the ADSC coating, this can hopefully stimulate faster and improved bone regrowth over time. Future studies will investigate in depth the in vivo efficacy of this potentially beneficial strategy.

\section{Conclusion}

In conclusion, the experiments presented here show that hydrothermally derived $\beta$-TCP can be seeded and coated with ADSCs by means of a common laboratory centrifuge at a higher number compared with static seeding, while still retaining the cell activity levels. This quick and easy method for homogenous cell coverage of scaffolds thereby enhances the therapeutic efficacy of the scaffold material.

\section{Acknowledgment}

The authors gratefully acknowledge the support of University of Technology Sydney Chancellor Post-Doctoral Research Fellowship.

\section{Author Disclosure Statement}

The authors declare no conflict of interest and no competing financial interests exist.

\section{References}

1. Langer RS, Vacanti JP. Tissue engineering: the challenges ahead. Sci Am. 1999;280:86-89.

2. Roy DM, Linnehan SK. Hydroxyapatite formed from coral skeletal carbonate by hydrothermal exchange. Nature. 1974; 247:220-222.

3. Ben-Nissan B. Natural bioceramics: from coral to bone and beyond. Curr Opin Solid State Mater Sci. 2003;7:283-288.

4. Chou J, Ben-Nissan B, Green DW, et al. Targeting and dissolution characteristics of bone forming and antibacterial drugs by harnessing the structure of micro-spherical shells from coral beach sand. Adv Eng Mater. 2011;13:93-99.

5. Li Y, Ma T, Kniss DA, et al. Effects of filtration seeding on cell density, spatial distribution, and proliferation in nonwoven fibrous matrices. Biotechnol Prog. 2001;17:935-944.

6. Kitagawa T, Yamaoka T, Iwase R, Murakami A. Threedimensional cell seeding and growth in radial-flow perfusion bioreactor for in vitro tissue reconstruction. Biotechnol Bioeng. 2006;93:947-954.

7. Cheng G, Youssef BB, Markenscoff P, Zygourakis K. Cell population dynamics modulate the rates of tissue growth processes. Biophys J. 2006;90:713-724.

8. Van Den Dolder J, Bancroft GN, Sikavitsas VI, et al. Flow perfusion culture of marrow stromal osteoblasts in titanium fiber mesh. J Biomed Mater Res. 2003;64A:235-241.

9. Dunkelman NS, Zimber MP, LeBaron RG, et al. Cartilage production by rabbit articular chondrocytes on polyglycolic acid scaffolds in a closed bioreactor system. Biotechnol Bioeng. 1995;46:299-305.

10. Sittinger M, Bujia J, Minuth WW, et al. Engineering of cartilage tissue using bioresorbable polymer carriers in perfusion culture. Biomaterials. 1994;15:451-456.

11. Green DW, Li G, Milthorpe B, Ben-Nissan B. Adult stem cell coatings using biomaterials for regenerative medicine. Mater Today. 2012;15:61-68.

12. Bunnell BA, Flaat M, Gagliardi C, et al. Adipose-derived stem cells: isolation, expansion and differentiation. Methods. 2008;45:115-120.

13. Vunjak-Novakovic G, Freed LE, Langer R. Effects of mixing on the composition and morphology of tissue-engineered cartilage. AIChE J. 1996;42:850-860.

14. Botchwey EA, Dupree MA, Pollack SR, et al. Tissue engineered bone: measurement of nutrient transport in threedimensional matrices. J Biomed Mater Res A. 2003;67:357-367.

Address correspondence to: Joshua Chou, PhD Advanced Tissue Regeneration and Drug Delivery Group School of Medical and Molecular Biosciences University of Technology Sydney P.O. Box 123

Ultimo, Sydney, NSW Australia

E-mail: joshua.chou@uts.edu.au 